\title{
Familial hypercholesterolaemia in South Africa: A reminder
}

It is ironic that South Africa (SA), known for the high prevalence of familial hypercholesterolaemia $(\mathrm{FH})^{[1]}$ in several population groups due to multiple founder effects, is lagging behind much of the world in the provision of effective and affordable preventive treatment to persons with $\mathrm{FH}$. FH is the most common serious genetic disorder in man and should be managed at a primary healthcare level. Treatment is safe and saves lives. The difficulties in managing $\mathrm{FH}$ in $\mathrm{SA}^{[2]}$ are also experienced elsewhere: there is a global call to action. ${ }^{[3]}$

$\mathrm{FH}$ is an autosomal dominant disorder with a gene dose effect manifesting as heterozygous (heFH) or homozygous (hoFH) phenotypes. Diagnostic criteria can be found in the SA guideline for dyslipidaemia ${ }^{[4]} \mathrm{HeFH}$ can be diagnosed confidently in the presence of an Achilles tendon xanthoma and a total cholesterol concentration of $>7.5 \mathrm{mmol} / \mathrm{L}$ without hypertriglyceridaemia. Tendon xanthomata, however, are not found in all patients with heFH and are uncommon before adulthood. Patients with severe hypercholesterolaemia and a family history of premature atherosclerotic cardiovascular disease or severe hypercholesterolaemia can be diagnosed with 'probable heFH'. HoFH usually presents in childhood, with cutaneous and tendinous xanthomata and hypercholesterolaemia $>13 \mathrm{mmol} / \mathrm{L}$.

$\mathrm{FH}$ is characterised biochemically by marked elevation of lowdensity lipoprotein cholesterol (LDL-C) due to impaired clearance, which is mostly attributable to dysfunction of the LDL-receptor (LDLR). However, its ligand, apolipoprotein B, may be defective, or proprotein convertase subtilisin/kexin type 9 (PCSK9), through gain of function mutations, results in excessive LDLR degradation. Nephrotic syndrome and hypothyroidism should always be excluded as secondary causes of hypercholesterolaemia. Ideally, the clinical diagnosis of FH should be confirmed by genetic testing, but as the aim of treatment is the control of LDL-C, genetic testing is not required prior to starting treatment.

FH affects about 1 in 300 persons $^{[5]}$ worldwide, but is more common in founder populations. In SA, these include white Afrikaans-speaking individuals, Jews and Indians (from Gujarat) with characteristic founder mutations; but many more mutations are known. ${ }^{[6]} \mathrm{FH}$ poses a very high risk of atherosclerosis and requires no additional risk factors for atherosclerotic cardiovascular disease to develop. A study in Cape Town revealed that untreated heFH patients on average experienced angina pectoris or myocardial infarction at the age of 45 years and died at an average age of 48 years. ${ }^{[7]}$

The therapeutic nihilism before the advent of statins impacted negatively on the management of FH in SA and has unfortunately not been completely overcome, despite good evidence for the benefit of statins. In Dutch children with heFH, prescription of statins improved cardiovascular prognosis compared with their affected parents. ${ }^{[8]}$ High doses of atorvastatin and rosuvastatin lower LDL-C by half, and adding ezetimibe to statins lowers the LDL-C by an additional $20 \%$. Such treatment will suffice in many persons with heFH to achieve recommended targets of LDL-C $(<1.8 \mathrm{mmol} / \mathrm{L}$ when additional risk factors are absent or $<1.4 \mathrm{mmol} / \mathrm{L}$ with an additional major risk factor or clinical atherosclerosis) ${ }^{[9]}$ Additional reduction of LDL-C by $\sim 50-60 \%$ by monoclonal antibodies directed against PCSK9 (alirocumab and evolocumab) is now available in SA, but is costly.

What is the scale of the problem of FH in SA? The number of affected persons likely exceeds 200 000. Given SA's demography, most persons with FH are black. Assuming that $80 \%$ of untreated FH persons will experience coronary disease before the age of 60 years, there are 160000 at-risk persons in whom early treatment could save many life-years. The exact cost benefit of prevention is difficult to assess. The Council for Medical Schemes ${ }^{[10]}$ reported that in 2019 the average cost of an admission for a myocardial infarction was approximately ZAR132 000 in the private sector. An appropriate statin currently costs $\sim$ ZAR2 400 annually at the single exit price ${ }^{[11]}$ and simply extrapolated over 40 years, costs ZAR96 000. Importantly, persons with FH may have several cardiovascular events.

All persons with FH should be identified and treated in primary healthcare, but more severe cases, e.g. patients with hypercholesterolaemia of $>12 \mathrm{mmol} / \mathrm{L}$, are at particularly high risk and require more comprehensive assessment and additional treatment. Some rare disorders (e.g. phytosterolaemia) requiring different therapeutic approaches may mimic FH. Unfortunately, training in SA in primary healthcare, internal medicine, endocrinology, cardiology, paediatrics and pathology often fails to provide meaningful exposure to patients with severe dyslipidaemias. Specialised lipid clinics therefore play an important role but are only readily accessible to patients in Cape Town and Johannesburg. Currently, there are only two public sector physicians with lipidology experience working part-time in lipid clinics. There is no dedicated laboratory service available for work-up of complex cases and no formal system of cascade screening following case identification. Ideally, patients with severe dyslipidaemia, estimated at $~ 500000$ in SA, would be managed equitably and fairly in a new unified healthcare system, irrespective of whether they come from the public or private sector. This service would be led by lipidologists. Lipidology is increasingly an avenue of specialisation in many countries. For many reasons, there seems to be no prospect of formally establishing such a discipline in SA.

The Lipid and Atherosclerosis Society of Southern Africa (LASSA) recently reminded the National Department of Health of the importance of FH. However, existing guidelines can still not be implemented due to a lack of funding in both the public and the private healthcare sectors. LASSA recently communicated a mechanism to medical schemes to expedite assessment of patients with severe dyslipidaemia, providing an informed opinion and diagnostic support for those who are in most urgent need for aggressive treatment. Persons with FH deserve fair treatment with medication comparable to the treatment provided for other seriously life-threatening conditions. Further to the steps taken by LASSA, an advocacy group may be required to improve detection and treatment for FH in SA.

\section{A D Marais}

Division of Chemical Pathology, Department of Pathology, Faculty of Health Sciences, University of Cape Town, South Africa

david.marais@uct.ac.za

\section{J Blom}

Division of Lipidology, Department of Medicine, Faculty of Health Sciences, University of Cape Town, South Africa

\section{F J Raal}

Division of Endocrinology, Department of Medicine, Faculty of Health Sciences, University of the Witwatersrand, Johannesburg, South Africa On behalf of the Lipid and Atherosclerosis Society of Southern Africa

\footnotetext{
Rubinsztein DC, van der Westhuyzen DR, Coetzee GA. Monogenic primary hypercholesterolaemia in South Africa. S Afr Med J 1994;84(6):339-344.

Marais AD. Familial hypercholesterolaemia and its management in South Africa. Cardiovasc J Af 2019;30(5):247-248. https://doi.org/10.5830/CVJA-2019-054
} 
3. Wilemon KA, Patel J, Aguilar-Salinas C, et al. Reducing the clinical and public health burden of familial hypercholesterolemia. A global call to action. JAMA Cardiol 2020;5(2):217-229. https://doi. org/10.1001/jamacardio.2019.5173

4. Klug E, Raal FJ, Marais AD, et al. South African dyslipidaemia guideline consensus statement: 2018 update. A joint statement from the South African Heart Association (SA Heart) and the Lipid and update. A joint statement from the South African Heart Association (SA Heart) and the Lipid and
Atherosclerosis Society of Southern Africa (LASSA). S Afr Med J 2018;26;108(11b):973-1000. https:// Atherosclerosis Society of Southern Africa (
doi.org/10.7196/SAMJ.2018.v108i11.13383

5. Hu P, Dharmayat KI, Stevens CAT, et al. Prevalence of familial hypercholesterolemia among the general population and patients with atherosclerotic cardiovascular disease. A systematic review and meta-analysis. Circulation 2020;141(22):1742-1759. https://doi.org/10.1161

6. Marais AD, Kotze MJ, Raal FJ, Khine AA, Talmud P, Humphries SE. Familial hypercholesterolaemia workshop for leveraging point-of-care testing and personalised medicine in association with the Lipic and Atherosclerosis Society of Southern Africa. Cardiovasc J Afr 2019;30(4):261-268. https://doi. org/10.5830/CVJA-2019-055
7. Firth JC, Marais AD. Familial hypercholesterolaemia: The Groote Schuur Hospital Experience. S Afr Med J 2008;98(2):99-104

8. Luirink IK, Wiegman A, Kusters DM, et al. 20-year follow-up of statins in children with familial hypercholesterolemia. N Engl J Med 2019;381(16):1547-1556. https://doi.org/10.1056/NEJMoa1816454 9. Klug EQ, Raal FJ. New cholesterol targets for patients at high or very high cardiovascular risk and the 9. Klug EQ, Raal FJ. New cholesterol targets for patients at high or very high cardiovascular risk and the
indications for PCSK9 inhibitors. S Afr Med J 2020;110(11):1059. https://doi.org/10.7196/SAMJ.2020. indications for

10. Council for Medical Schemes. For the benefit of the member. Annual Report 2019/20. https://www. medicalschemes.co.za/annualreport2020/ (accessed 4 June 2021).

11. Medicine Price Registry | Health-e. https://health-e.org.za/medicine-price-registry/ (accessed 13 April 2021).

S Afr Med J 2021;111(8):700-701. https://doi.org/10.7196/SAMJ.2021.v111i8.15782 